\title{
The potential of GHK as an anti-aging peptide
}

\author{
Yan Dou ${ }^{\mathrm{a}}$, Amanda Lee ${ }^{\mathrm{a}}$, Lida Zhu ${ }^{\mathrm{a}}$, John Morton ${ }^{\mathrm{a}}$, Warren Ladiges ${ }^{\mathrm{a},{ }^{*}}$ \\ ${ }^{a}$ Department of Comparative Medicine, School of Medicine, University of Washington, Seattle, WA, USA.
}

\begin{abstract}
GHK (glycyl-L-histidyl-L-lysine) is a naturally occurring peptide found in human serum with levels averaging $200 \mathrm{ng} / \mathrm{ml}$ at age 20 but declining to an average of $80 \mathrm{ng} / \mathrm{ml}$ by age 60 . The molecule has a very high affinity for copper and forms the chelate GHK-Cu. The peptide as well as its $\mathrm{Cu}$ (II) chelate have anti-inflammatory and tissue remodeling properties. GHK-Cu has been shown to promote skin remodeling, wound healing and regeneration, and has prominent antioxidant and anti-inflammatory effects in in vitro and in vivo studies. In addition, preliminary observations suggest GHK can partially reverse cognitive impairment in aging mice by targeting anti-inflammatory and epigenetic pathways. The evidence as presented provides the rationale to further investigate this naturally occurring peptide in preclinical and clinical aging studies.
\end{abstract}

Keywords: GHK peptide, GHK-Cu chelate, anti-aging, antioxidant, anti-inflammatory, age-related cognitive impairment

\section{What is GHK?}

GHK (glycyl-L-histidyl-L-lysine) is a naturally occurring peptide originally found in human serum and shown to stimulate growth in hepatoma cells [1]. The molecule has a very high affinity for copper (II) and forms the chelate GHK-Cu. The plasma level of GHK is about $200 \mathrm{ng} / \mathrm{ml}$ at age 20 but declines to $80 \mathrm{ng} / \mathrm{ml}$ by age 60 . However, no studies have yet been reported linking low serum GHK levels with specific aging processes or age-related disease. The peptide is cleaved and released from its parent protein SPARC during the degradation of extracellular matrix, to aid in tissue remodeling by increasing levels of angiogenesis [2]. Both GHK and GHK-Cu have been shown to have anti-inflammatory and tissue remodeling properties. GHK-Cu has been studied in clinical research showing positive effects on skin remodeling and regeneration.

\section{The tissue remodeling effect of GHK}

GHK-Cu has been extensively studied for its tissue re-

\footnotetext{
* Corresponding author: Warren Ladiges

Mailing address: Department of Comparative Medicine, School of Medicine, University of Washington, Seattle, WA 98195, USA. Email: wladiges@uw.edu
}

Received: 06 March 2020 / Accepted: 09 March 2020 modeling and wound healing abilities for nearly four decades [3]. GHK-Cu at a concentration of $1 \mathrm{nM}$ increases the expression of basic fibroblast growth factor (bFGF) and vascular endothelial growth factor (VEGF) in irradiated human dermal fibroblasts, both of which aid blood vessel formation and blood flow into damaged tissues [46]. It also increases HUVECs proliferation by stimulating VEGF and FGF-2 expressions, promoting angiogenesis that aids in wound healing [7]. GHK-Cu at low concentrations is a powerful attractant for capillary cells that build new blood vessels, and a powerful migration stimulant for macrophages and mast cells that remove damaged cellular debris and secrete proteins important for wound contraction and tissue healing [8-9]. Since copper is an important trace element that plays a key factor in the production of collagen and elastin, GHK-Cu can be a natural supplement for copper in the connective tissue synthesis [10-11]. It is also involved in the activation of the synthesis of matrix compounds in vivo, which are critical for the formation of a resistant new tissue [12-13].

Moreover, GHK-Cu promotes bone healing and enhances osteoblastic cell attachment, potentially through the increase in collagen synthesis [14-15]. Articular injection of $0.3 \mathrm{mg} / \mathrm{ml} \mathrm{GHK}-\mathrm{Cu}$ enhances some of the healing outcomes in a rat model of anterior cruciate ligament reconstruction (ACLR), and the treatment of GHK-Cu encapsulated in liposome significantly fasten burn wound healing in mice with scald wound $[7,16]$. In addition to wound healing studies in mice, clinical studies have been done on the effect of GHK-Cu on skin regeneration, which show a more rapid healing process and better skin conditions with 


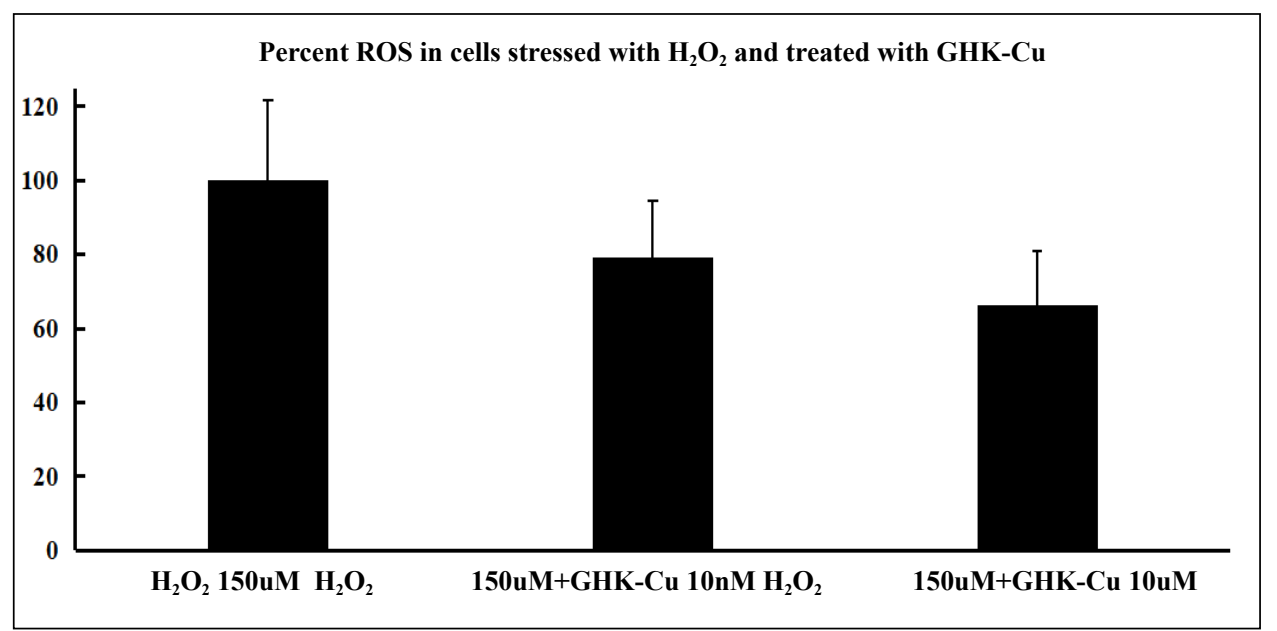

Figure 1. ROS level was measured in WI-38 cells treated with $150 \mathrm{uM}$ hydrogen peroxide $\left(\mathrm{H}_{2} \mathrm{O}_{2}\right)$, with or without pretreatment of GHK-Cu. ROS readout is shown in the $\mathrm{y}$-axis as percentage, with $\mathrm{H}_{2} \mathrm{O}_{2} 150 \mathrm{uM}$ group being 100 percent. Cells pretreated with GHK$\mathrm{Cu}$ at a concentration of $10 \mathrm{nM}$ and $10 \mathrm{uM}$ showed significant decrease in ROS. All data represent the mean \pm SEM, $n=6$. $* * P<0.01, * P$ $<0.05$, statistically significant difference between GHK-Cu treated cells and control cells.

GHK-Cu in the skin cream $[3,17]$.

\section{The anti-inflammatory effect of GHK}

Both GHK and its copper (II) complex GHK-Cu have been studied for antioxidant and anti-inflammatory activities. Without being bound to copper, GHK has been shown to be a quencher of cytotoxic end products of lipid peroxidation such as $\alpha, \beta-4$-hydroxy-trans-2-nonenal and acrolein, which can be associated with severe pathologies such as diabetes and neurodegenerative disease [1819]. With a concentration as low as $10 \mathrm{uM}$, the addition of GHK can reduce the tert-butyl hydroperoxide-induced reactive oxygen species (ROS) level in Caco-2 cells by almost 50-percent. Moreover, GHK has been shown to be a quencher for hydroxyl and peroxyl radicals by ESR spectroscopy, with its ability to quench hydroxyl radicals much stronger than glutathione (GSH), making it a strong endogenous antioxidant [20]. In a bleomycin-induced fibrosis mouse model, treatment with GHK showed reduced inflammatory cell infiltration and interstitial thickness with reduced TNF- $\alpha$ and IL-6 expression, suggesting GHK as a potential treatment for pulmonary fibrosis [21]. Compared with GHK, its copper (II)-chelated form shows a more prominent antioxidant and anti-inflammatory effect in both in vitro and in vivo studies. Pretreatment of RAW 264.7 macrophage cells with GHK-Cu has been shown to significantly decrease ROS levels induced by lipopolysaccharide (LPS), increase levels of SOD activities and total GSH, and decrease levels of TNF- $\alpha$ and IL- 6 production through the suppression of NF- $\mathrm{kB}$ p65 and p38 MAPK signaling [22]. Both signaling pathways are key regulators of inflammation and pro-inflammatory responses, which are considered targets for developing anti-inflammatory therapeutic agents [23-26]. In the LPS-induced acute lung injury mouse model, treatment of GHK-Cu also significantly suppressed TNF- $\alpha$ and IL- 6 expression with a significant decrease of both NF-kB p65 and p38 MAPK signaling, suggesting that $\mathrm{GHK}-\mathrm{Cu}$ is useful for the development of novel anti-inflammatory therapies [22]. We have also found that pretreatment of GHK-Cu has a robust antioxidant effect in WI-38 cells treated with $150 \mathrm{uM}$ of hydrogen peroxide, decreasing the ROS level to almost 60 percent (Figure 1).

\section{GHK partially reverses age-related cognitive impairment in mice}

Since GHK is absorbed at a high efficiency across the blood brain barrier into the brains of rodents and nonhuman primates when delivered parenterally, testing the effects of the peptide on neurological function is warranted. C57BL/6 male mice, 28 months of age, were treated with GHK at a dose of $10 \mathrm{mg} / \mathrm{kg}$ body weight 5 times per week for three weeks. Control mice were treated with saline in a similar manner. Mice treated with GHK were able to find the escape hole significantly faster in trials 4 and 5 compared to mice treated with saline (Figure 2) as assessed by a Box Maze spatial navigation learning task [27] at the end of the treatment period. Immunohistochemistry of brain tissues from mice treated with GHK showed evi-

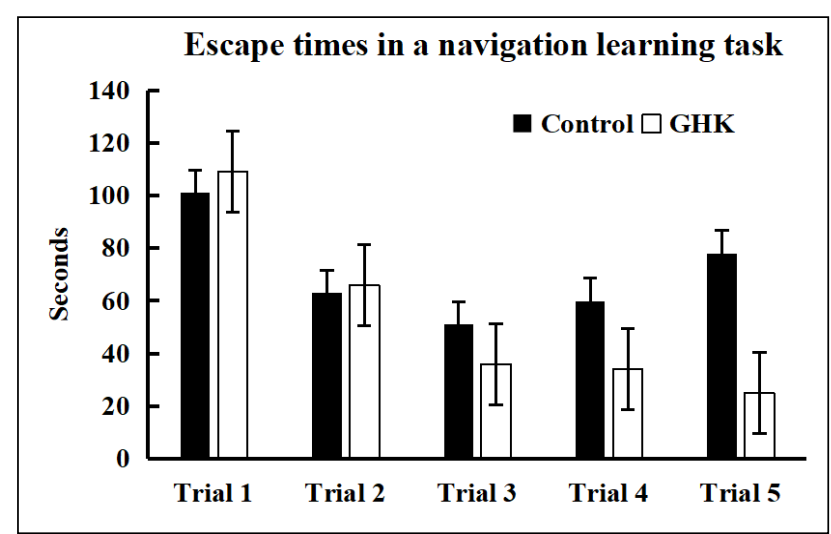

Figure 2. C57BL/6 male mice, 28 months of age, treated with GHK at a dose of $10 \mathrm{mg} / \mathrm{kg}$ body weight 5 times per week for 3 weeks showed improved learning compared to saline treated control mice. Data represent the mean $\pm \mathrm{SEM}, n=10$ /cohort, $* P<0.05$ statistically significant difference. 
dence of decreased inflammation and increased labeling of histone deacetylase 2, suggesting that in addition to an anti-inflammatory effect, GHK may be triggering an epigenetic pathway in the amelioration of cognitive impairment in aging mice.

\section{Summary and future directions}

The ability of GHK and its $\mathrm{Cu}$ chelate to promote skin remodeling, wound healing and regeneration, and its prominent antioxidant and anti-inflammatory effects are qualities that will help enhance healthy aging [28-31]. In addition, the novel observation that GHK might target an epigenetic pathway further enhances its potential as an anti-aging peptide [32]. The evidence as presented provides the rationale to further investigate this naturally occurring peptide in preclinical and clinical aging studies. The benefits of GHK in terms of long-term effects on slowing aging could have a high impact on increasing the quality of life with increasing age. There is also the possibility that GHK could be extremely useful combined with other antiaging drugs as a cocktail [33] to robustly enhance healthy aging.

\section{Declarations}

Acknowledgements: This work was supported in part by NIA grant R01 AG057381 (Ladiges, PI).

Conflict of Interest: Warren Ladiges is a member of the Editorial Board of Aging Pathobiology and Therapeutics. All authors declare no conflict of interest and were not involved in the journal's review or desicions related to this manuscript.

\section{References}

1. Pickart L, Thayer L, Thaler M M. A synthetic tripeptide which increases survival of normal liver cells, and stimulates growth in hepatoma cells. Biochemical and biophysical research communications, 1973, 54(2): 562566.

2. Lane T F, Iruela-Arispe M L, Johnson R S, et al. SPARC is a source of copper-binding peptides that stimulate angiogenesis. The Journal of cell biology, 1994, 125(4): 929943.

3. Pickart L. The human tri-peptide GHK and tissue remodeling. Journal of Biomaterials Science, Polymer Edition, 2008, 19(8): 969-988.

4. Pollard J D, Quan S, Kang T, et al. Effects of copper tripeptide on the growth and expression of growth factors by normal and irradiated fibroblasts. Archives of facial plastic surgery, 2005, 7(1): 27-31.

5. Steiling H, Werner S. Fibroblast growth factors: key players in epithelial morphogenesis, repair and cytoprotection. Current opinion in biotechnology, 2003, 14(5): 533-
537.

6. Powers C J, McLeskey S W, Wellstein A. Fibroblast growth factors, their receptors and signaling. Endocrine-related cancer, 2000, 7(3): 165-197.

7. Wang X, Liu B, Xu Q, et al. GHK-Cu-liposomes accelerate scald wound healing in mice by promoting cell proliferation and angiogenesis. Wound Repair and Regeneration, 2017, 25(2): 270-278.

8. Raju K S, Alessandri G, Gullino P M. Characterization of a chemoattractant for endothelium induced by angiogenesis effectors. Cancer research, 1984, 44(4): 1579-1584.

9. Poole T J, Zetter B R. Stimulation of rat peritoneal mast cell migration by tumor-derived peptides. Cancer research, 1983, 43(12 Part 1): 5857-5861.

10. Maquart F X, Bellon G, Chaqour B, et al. In vivo stimulation of connective tissue accumulation by the tripeptidecopper complex glycyl-L-histidyl-L-lysine-Cu2+ in rat experimental wounds. The Journal of clinical investigation, 1993, 92(5): 2368-2376.

11. Siegel R C, Pinnell S R, Martin G R. Cross-linking of collagen and elastin. Properties of lysyl oxidase. Biochemistry, 1970, 9(23): 4486-4492.

12. Siméon A, Monier F, Emonard H, et al. Expression and activation of matrix metalloproteinases in wounds: modulation by the tripeptide-copper complex glycyl-Lhistidyl-L-lysine-Cu2+. Journal of investigative dermatology, 1999, 112(6): 957-964.

13. Siméon A, Wegrowski Y, Bontemps Y, et al. Expression of glycosaminoglycans and small proteoglycans in wounds: modulation by the tripeptide-copper complex glycyl-lhistidyl-l-lysine-Cu2+. Journal of Investigative Dermatology, 2000, 115(6): 962-968.

14. Pohunkova H, Stehí J, Vachal J, et al. Morphological features of bone healing under the effect of collagen-graftglycosaminoglycan copolymer supplemented with the tripeptide Gly-His-Lys. Biomaterials, 1996, 17(16): 1567-1574.

15. Godet D, Marie P J. Effects of the tripeptide glycyl-L-histidyl-L-lysine copper complex on osteoblastic cell spreading, attachment and phenotype. Cellular and molecular biology (Noisy-le-Grand, France), 1995, 41(8): 10811091.

16. Fu S C, Cheuk Y C, Chiu W Y V, et al. Tripeptide-copper complex GHK-Cu (II) transiently improved healing outcome in a rat model of ACL reconstruction. Journal of Orthopaedic Research, 2015, 33(7): 1024-1033.

17. Pickart L, Vasquez-Soltero J M, Margolina A. GHK peptide as a natural modulator of multiple cellular pathways in skin regeneration. BioMed research international, 2015, 2015.

18. Beretta G, Artali R, Regazzoni L, et al. Glycyl-histidyllysine (GHK) is a quencher of $\alpha, \beta$-4-hydroxy-trans-2nonenal: a comparison with carnosine. Insights into the mechanism of reaction by electrospray ionization mass spectrometry, $1 \mathrm{H} \mathrm{NMR}$, and computational techniques. Chemical research in toxicology, 2007, 20(9): 13091314.

19. Beretta G, Arlandini E, Artali R, et al. Acrolein sequester- 
ing ability of the endogenous tripeptide glycyl-histidyllysine (GHK): characterization of conjugation products by ESI-MSn and theoretical calculations. Journal of pharmaceutical and biomedical analysis, 2008, 47(3): 596602.

20. Sakuma S, Ishimura M, Yuba Y, et al. The peptide glycylL-histidyl-L-lysine is an endogenous antioxidant in living organisms, possibly by diminishing hydroxyl and peroxyl radicals. International journal of physiology, pathophysiology and pharmacology, 2018, 10(3): 132.

21. Zhou X M, Wang G L, Wang X B, et al. GHK peptide inhibits bleomycin-induced pulmonary fibrosis in mice by suppressing TGF $\beta 1 /$ Smad-mediated epithelial-to-mesenchymal transition. Frontiers in Pharmacology, 2017, 8: 904.

22. Park J R, Lee H, Kim S I, et al. The tri-peptide GHK-Cu complex ameliorates lipopolysaccharide-induced acute lung injury in mice. Oncotarget, 2016, 7(36): 58405.

23. Gao M, Chen L, Yu H, et al. Diosgenin down-regulates NF$\kappa \mathrm{B}$ p65/p50 and p38MAPK pathways and attenuates acute lung injury induced by lipopolysaccharide in mice. International immunopharmacology, 2013, 15(2): 240245.

24. Liu T, Zhang L, Joo D, et al. NF- $\kappa B$ signaling in inflammation. Signal transduction and targeted therapy, 2017, 2(1): 1-9.

25. Kaminska B. MAPK signalling pathways as molecular targets for anti-inflammatory therapy-from molecular mechanisms to therapeutic benefits. Biochimica et Biophysica Acta (BBA)-Proteins and Proteomics, 2005,
1754(1-2): 253-262.

26. Yong H Y, Koh M S, Moon A. The p38 MAPK inhibitors for the treatment of inflammatory diseases and cancer. Expert opinion on investigational drugs, 2009, 18(12): 1893-1905.

27. Mukherjee K, Lee A, Zhu L, et al. Sleep-deprived cognitive impairment in aging mice is alleviated by rapamycin. Aging Pathobiology and Therapeutics, 2019, 1(1): 05-09.

28. Gould L, Abadir P, Brem H, et al. Chronic wound repair and healing in older adults: current status and future research. Wound Repair and Regeneration, 2015, 23(1): 1-13.

29. Davalli P, Mitic T, Caporali A, et al. ROS, cell senescence, and novel molecular mechanisms in aging and age-related diseases. Oxidative medicine and cellular longevity, 2016, 2016.

30. McHugh D, Gil J. Senescence and aging: Causes, consequences, and therapeutic avenues. Journal of Cell Biology, 2018, 217(1): 65-77.

31. Santos A L, Sinha S, Lindner A B. The good, the bad, and the ugly of ROS: New insights on aging and aging-related diseases from eukaryotic and prokaryotic model organisms. Oxidative medicine and cellular longevity, 2018, 2018.

32. Pasyukova E G, Vaiserman A M. HDAC inhibitors: A new promising drug class in anti-aging research. Mechanisms of ageing and development, 2017, 166: 6-15.

33. Sharma K C, Wang J, Jiang Z, et al. The rationale for testing drug combinations in aging intervention studies. Aging Pathobiology and Therapeutics, 2019, 1(1): 01-04.

Cite this article as: Dou Y, Lee A, Zhu L, et al. The potential of GHK as an anti-aging peptide[J]. Aging Pathobiology and Therapeutics, 2020, 2(1): 58-61. 